\title{
HOT GAS FLOWS IN ELLIPTICAL GALAXIES
}

\author{
ALVIO RENZINI \\ European Southern Observatory \\ Garching bei München, Germany
}

\section{Introduction}

Stars in elliptical galaxies lose mass at an overall present rate $\dot{M}_{*} \simeq 1.5 \times$ $10^{-11} L_{B} \quad M_{\odot} y r^{-1}$ (e.g., Faber \& Gallagher 1976; Renzini \& Buzzoni 1986). When allowing for the predicted increase back with cosmological time it turns out that over one Hubble time the stellar population of an elliptical galaxy has cumulatively lost $20-50 \%$ of its initial mass, the precise value depending on the IMF. This review focuses on two simple questions: what happens to the gas being lost by the stars? Where is it ultimately disposed?

Ellipticals are dynamically hot stellar systems, hence the cool stellar winds emanating from individual red giants are quickly heated to nearly the same dynamical temperature of the stars, as such winds collide with each other of with the ambient ISM. This implies $T \simeq m_{p} \sigma_{\star}{ }^{2} / k \simeq 10^{7} \mathrm{~K}$ ( $\sigma_{\star}$ being the stellar velocity dispersion), and the gas will shine in $\mathrm{X}$ rays.

Fig. 1 shows the X-ray luminosity of the elliptical galaxies vs their optical luminosity. Data refer to the objects observed with the Einstein satellite that have been recently reanalysed in a homogeneous way by Fabbiano, Kim, \& Trinchieri (1992). Galaxies at any given optical luminosity exhibit a very wide range of $\mathrm{X}$-ray luminosities, and Fig.2 demonstrates that this is not a result of distance errors (i.e. this is a distance-independent diagram). So, galaxies with the same luminosity and/or the same $\sigma_{\star}$ may differ by up to a factor $\sim 100$ in their X-ray luminosity.

The lines in Fig. 1 show the predicted X-ray luminosity for the two classical stady state solutions for the gas flow. In a steady state supersonic wind (Mathews \& Baker 1971) very little hot gas accumulates, and $L_{X}$ reduces to $\sim L_{d i s c r}$, the contribution of discrete sources. In a steady state "cooling flow" (e.g. Sarazin \& White 1987) a dense ISM is in place, and $L_{X} \simeq L_{i n}$. Clearly, neither of the two steady state solutions accounts for the 


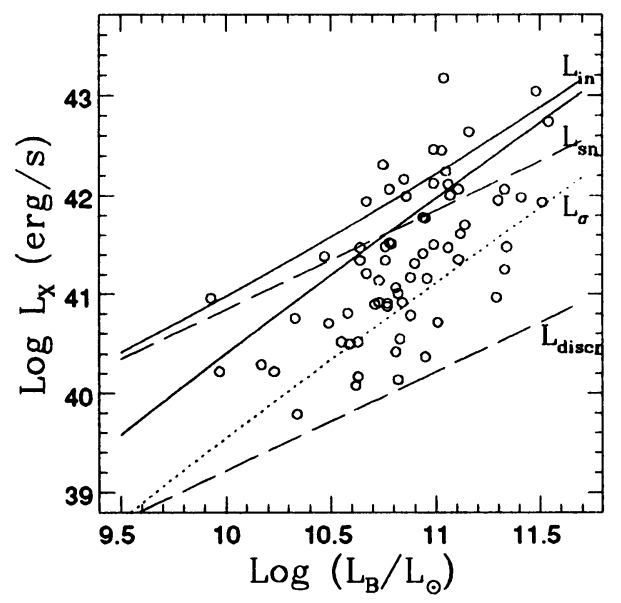

Figure 1. The X-ray luminosity of elliptical galaxies observed with the Einstein Observatory vs their optical luminosity (From Fabbiano et al. 1992). $L_{d i s c r}, L_{\sigma}$ and $L_{s n}$ are the expected contributions from discrete sources, from the thermalization of stellar winds, and from $\mathrm{SN}$ explosions (with $\vartheta_{S N}=1$ ), respectively. The expected X-ray luminosity of galaxies in the inflow regime $\left(L_{i n}\right)$ is also shown, for $\vartheta_{S N}=0$ and 1 (adapted from Ciotti et al. 1991).

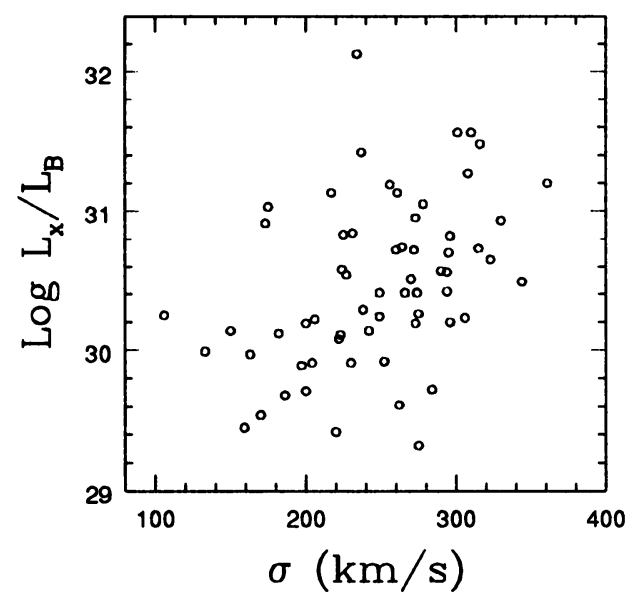

Figure 2. The X-ray to optical luminosity ratio for the elliptical galaxies in Fig. 1 as a function of the central velocity dispersion. Luminosity units are as in Fig. 1.

observed X-ray properties of the galaxies. Some galaxies are faint enough in $\mathrm{X}$-rays for their gas flow to be in a supersonic wind regime, and indeed their $\mathrm{X}$-ray spectrum requires very little hot ISM contribution in excess of the emission from low mass X-ray binaries and other stellar sources (Pellegrini \& Fabbiano 1994). Seemingly, some galaxies are bright enough in X-rays for being in a cooling flow regime. However, most galaxies exhibit X-ray luminosities that are intermediate between these two extremes. 
By postulating a distibuted sink of gas from the hot ISM (e.g., Sarazin \& Ashe 1989; Bertin \& Toniazzo 1995), the X-ray luminosity of cooling flow models could be somewhat reduced, as only part of the flow would reach the center. Yet not enough to account for the galaxies for which $L_{X}<L_{s n}+L_{\sigma}$ (where $L_{s n}$ and $L_{\sigma}$ are the rates of energy deposition from supernova explosions and from the thermalization of stellar winds, respectively). Thus, steady state models of any kind can hardly account for the observed distribution of X-ray luminosities. There are also good theoretical reasons for dismissing steady state solutions. Indeed, both the rate of stellar mass return and the supernova (SN) heating are expected to exhibit a strong secular evolution, and therefore evolutionary - as opposed to time-independent - solutions are to be sought.

Life may get rather complicated when steady state solution are abandoned. Besides a time-dependent rate of stellar mass return a cornucopia of other time-dependent ingredients are likely to play a role, along with the very many parameters needed to describe them (see Renzini 1993 for a review). These include the evolution of the $\mathrm{SN}$ heating, the possible occurrence of star formation, the role of the intracluster medium (ram)pressure, recuTrent local thermal instabilities (Kritsuk 1992), and the interactions of the gas flow with a massive black hole (BH) that may exist at the center of galaxies. Here I concentrate on the role of two such ingredients, $\mathrm{SN}$ heating and $\mathrm{BH}$-gas flow interactions.

\section{What role for Type Ia Supernovae?}

Evolutionary models of gas flows that include dark matter and SN heating meet several of the observed X-ray properties of ellipticals. There are basically two families of models, depending on the assumed past evolution of the Type Ia SN heating, that can be conveniently parameterized assuming a power-law evolution with time (Rate $\propto t^{-s}$ ). Also the evolution of the rate of stellar mass return is well represented by a power-law, with $\dot{M}_{\star} \propto \sim t^{-1.3}$ for a Salpeter IMF. Hence, for $s \gtrsim 1.3$ the specific heating of the stellar ejecta secularly decreases with time (the SN heating was more efficient in the past). (Galaxy flows correspondingly evolve from early supersonic winds, to subsonic outflow, to an inflow regime when SN heating finally falls short of compensating for the radiative losses, and a central cooling catastrophe takes place (Ciotti et al. 1991, hereafter WOI models). Coversely, for $s \lesssim 1.3$ the specific SN heating increases with time, and flow models evolve in the reverse sequence of flow regimes, from early inflows, to subsonic outflows, to late supersonic winds (Loewenstein \& Mathews 1987; David, Forman, \& Jones 1991, hereafter IOW models).

The X-ray luminosity is very low during wind $(\mathrm{W})$ phases, as the hot 
gas rapidly leaves the galaxy and does not accumulate. During inflow (I) phases galaxies are very bright in X-rays, as the hot gas remains confined to the galaxy and cools down. During outflow $(\mathrm{O})$ phases $L_{X}$ is intermediate between the two previous extremes, increasing with time in the WOI models as gas accumulates, decreasing with time in the IOW models. Thus, the large dispersion in the $L_{X} / L_{B}$ ratio can be easily understood in the frame of either the WOI or the IOW models, and follows from relatively small differences in e.g. the depth of the potential well causing relatively large differences in the epoch of the O-I or I-O transition. In the frame of WOI models, in Fig. 1 galaxies evolve upward, from the lower dashed line when they are in the $\mathrm{W}$ phase, to the top line when they finally reach the I phase. IOW models instead drip from the top line, evolve downward, and finally land on the bottom line. In both cases, the observed X-ray luminosities indicate that most of present-day ellipticals are in the $O$ phase.

How to distinguish between these two opposite options? The best, most direct way would be to look at high redshift ellipticals, with $z \gtrsim 1$, hence $t \lesssim 5$ Gyr. In the case of IOW models high- $z \mathrm{E}$ galaxies would be brighter in X-rays compared to low- $z$ galaxies. while the reverse applies to WOI models. Unfortunately this is curerently unfeasible; e.g. ROSAT can hardly see individual galaxies beyond the distance of the Coma cluster.

The present and past rate of SNIa's in ellipticals being the crucial ingredients, these can be constrained by the observed elemental abundances in their ISM and in the intracluster medium (ICM; Ciotti et al. 1991; Renzini et al. 1993). Within galaxies, one expects the iron abundance in the hot ISM to be $\left.X_{F e}^{I S M} \simeq<X_{F e}^{*}\right\rangle+5 \vartheta_{S N}$, thus reflecting the average stellar abundance $\left(\left\langle X_{F e}^{*}\right\rangle\right)$ further enriched by Type Ia $S N_{s}, v_{S N}$ being their present rate in units of $2.2 \times 10^{-13} \mathrm{SNs} y r^{-1} L_{(:)}{ }^{-1}$. The most extreme estimates of the Type Ia $S N$ rate per unit light in ellipticals indicate $0.25 \lesssim \vartheta_{S N} \lesssim 1$ (Cappellaro, these proceedings), hence one expects $X_{F e}^{I S M} \gtrsim 2 X_{F e}^{(:)}$(Ciotti et al. 1991). From the iron-L complex at $\sim 1 \mathrm{keV}$ very low values are instead inferred from ASCA data, $X_{F e-L}^{I S M} \lesssim 0.3 X_{F e}^{(\cdot)}$ (Loewenstein et al. 1994, Matsushita, these proceedings). Formally, the ASCA results requires a vanishing SNIa activity in present day ellipticals. This Iron-L Discrepancy is extensively discussed by Arimoto (these proceedings).

The total amount of iron in clusters of galaxies (ICM plus galaxies) represent a record of overall past $\mathrm{SN}$ activity, hence it sets a constraint on the past evolution of the rate. The total iron mass to cluster optical luminosity ratio appears to be constant among clusters, irrespective of their luminosity, with $M_{F e} / L_{B} \simeq 0.03 \pm 0.01\left(M_{(:)} / L_{(:)}\right)$. To make all this iron requires either a much stronger SNla activity in the past $(s \gtrsim 1.3)$, or a much stronger Type II SN activity at early times than expected from a Salpeter IMF (Ciotti et al. 1991; Arnaud et al. 1992; Renzini et al. 1993; 
Elbaz, Arnaud, \& Vangioni-Flam 1995). The elemental abundances in the ICM offer an attractive way to distinguish between these two alternatives (Renzini $e t$ al. 1993), hence providing indirect constraints on the history of gas flows in ellipticals. Again, ASCA observations seem to require very little SNIa contribution to the ICM (Mushotzky et al. 1995), which in turn would favor IOW over WOI models. If confirmed, this result would reduce SNIa's to a very marginal role in the chemical evolution of galaxies at a galaxy cluster scale, thus rising the puzzling question as to how the solar elemental proportions have been established in our own Galaxy, while nonsolar proportions may prevail at galaxy cluster scales.

\section{Gas Flows and Central Black Holes}

If SNIa's are unimportant iron producers, they automatically become unimportant also as a heating source for galaxy gas flows (Ciotti et al. 1991). An alternative source of energy needs therefore to operate in order to power the outflows prevailing in present-day ellipticals. Nuclear activity seems to offer a very attractive option. If material being lost by stars is to flow to the center of a galaxy, then it can provide fuel to a central black hole $(\mathrm{BH})$ that may be lurking there (e.g. Gisler 1976). The idea has been recently explored and expanded by Ciotti \& Ostriker (1995), and a brief summary of some of their main results is given here.

During inflow phases $\sim 1-10 M_{(\mathrm{)})} y r^{-1}$ sink towards the center of the galaxy, and either IOW or WOI models simply postulate that this mass silently disappear. However, if instead this mass flow is accreted by a central $\mathrm{BH}$ the luminosity of the so powered $\mathrm{AGN}$ is expected to be in the range:

$$
L_{X}^{A G N} \simeq \eta \dot{M}_{S I N K} c^{2} \simeq 10^{45}-10^{47} \operatorname{erg~s}^{-1}
$$

for $0.01<\eta<0.1$, i.e., for 1 to $10 \%$ mass-energy conversion efficiency, with most of this luminosity being emitted in the hard X-ray range.

Moreover, during inflow phases a rather massive ISM is in place, and Ciotti \& Ostriker estimate an optical depth for the Compton scattering of hard X-ray photons $\tau^{I S M} \simeq 10^{-3}$. Therefore, the rate of energy deposition in the hot ISM is of the order of $\sim \tau^{I S M} L_{X}^{A G N} \simeq 10^{42}-10^{44} \mathrm{erg} \mathrm{s}^{-1}$, much larger than the radiative losses of the hot ISM (i.e., of the X-ray luminosity of the galaxy before the activation of the AGN, see Fig. 1). A strong feedback is then extected on the gas flow itself, and in the Ciotti \& Ostriker models the AGN energy deposition in the flow reverts the inflow back to a supersonic wind, thus discontinuing the fueling of the central $\mathrm{BH}$.

Correspondingly, the evolution of the gas flow proceeds through a series of outflow phases separated by brief AGN phases during which the ISM of the galaxy is expelled. After each expulsion the AGN dies out of starvation, 
and stellar mass loss starts replenishing the ISM again until the next central cooling catastrophe takes place. The duration of the outflow phases depends on several model parameters, and can be as long as several Gyr provided SN heating delays the central cooling catastrophe. Also the duration of the AGN phase is highly model dependent, typically several $10^{6} \mathrm{yr}$, leading to AGN phases duty cycle $\sim 10^{-3}$. These $1 \mathrm{D}$ models also predict high flickering activity during the active phases, as the $\mathrm{BH}$ accretion rate fluctuates and/or a transient cocoon of high optical depth $(\tau \simeq 1)$ builds up in the immediate vicinities of the $\mathrm{BH}$. The $\mathrm{X}$-ray luminosity evolution of the galaxies (taking out the AGN phases) is confined between the upper and the lower lines in Fig. 1, and therefore this model quite naturally accounts for the observed large dispersion of the $L_{X} / L_{B}$ ratios. After an AGN phase galaxies climb up slowly (in $\sim 1$ Gyr or more) starting from $L_{X} \simeq L_{\text {discr }}$, until the transition to the inflow takes place at $L_{X} \simeq L_{i n}$ and the AGN is activated, then precipitously falling back to $L_{X} \simeq L_{\text {discr }}$ before starting a new cycle.

An attractive feature of this model is that it accounts for the fate of the cooling gas during the inflow phases, an aspect that remains obscure in other models. Just $\lesssim 1 \%$ of the stellar ejecta eventually terminates inside the central $\mathrm{BH}$, thus releasing enough energy to eject the other $\sim 99 \%$ and enriching in metals the ICM. We may also wander if this same process could also work at the much larger scale of galaxy cluster "cooling flows", where a similar embarrassment exists as to where the cooling gas is eventually disposed. In other words, may central BHs in $\mathrm{cD}$ galaxies affect cluster scale "cooling flows"? Clearly a much brighter and hotter QSO would be required to compensate for radiative losses in cluster flows $\left(L_{X} \simeq 10^{45} \mathrm{erg}\right.$ $\mathrm{s}^{-1}$ ), and temporarily revert inflows to outflows near the cluster center.

Central BH-gas flow interactions may also take place at a much smaller scale. At the deep bottom of the potential well of ellipticals stellar, hence gas density and radiative losses can be very high, and mini-inflows inside $\sim 200 \mathrm{pc}$ are common while the rest of the galaxy is in a wind or outflow regime (Ciotti et al. 1991). If central massive BHs are ubiquitous among ellipticals, mini-inflows with typical mass deposition rates $\sim 10^{-3}-10^{-2} M_{(:)}$ $\mathrm{yr}^{-1}$ may feed a low-level, sporadic AGN activity, that may account for the observed $L_{R A D I O}-L_{X}$ correlation (see Fabbiano, these proceedings), and producing occasional flares at the center of elliptical galaxies. A $10^{6} L_{(:)}$ultraviolet flare that might have been generated in this way has been recentlty detected with HST at the very center of the elliptical galaxy NGC 4552 (Renzini et al. 1995), one of the X-ray brightest galaxies in Fig. 1. Future HST observations - especially in the ultraviolet - should tell us how common these flare events are, and whether their occurrence correlates with other optical and X-ray properties of the host galaxies. 


\section{Conclusions}

The study of gas flows in ellipticals is a fairly interesting field of research, as such flows connect to the central, active regions of galaxies to the one end, and to the intracluster medium at the other end, thus involving a rich variety of astrophysical phenomena. In summary, here stands the issue:

- Gas flows in ellipticals are not in a steady state.

- Outflows and winds appear to prevail over inflows in most ellipticals.

- The evolution of the flows over cosmological times is controlled by the evolution of the rate of Type Ia SNs, that is not yet constrained by direct observations.

- The iron and other element abundances in the ISM of ellipticals and in the ICM provide an indirect measure of the present and past SN rate, hence providing constraints for the evolution of the gas flows.

- Central BHs may play a main role in the evolution of the flows, with its interaction with the hot ISM leading potentially to a great variety of observable phenomena.

I am very grateful to Silvia Pellegrini for her preparation of the two figures shown in this paper and to her, Luca Ciotti and Annibale D'Ercole for stimulating discussions.

\section{References}

Arnaud, M., Rothenflug, R., Boulade, O., Vigroux, L., \& Vangioni-Flam, E. 1992, A\&A, 254, 49

Bertin, G., \& Toniazzo, T. 1995, ApJ, 451, 111

Ciotti, L., \& Ostriker, J.P. 1995, in preparation

Ciotti, L., D’Ercole, A., Pellegrini, S., \& Renzini, A. 1991, 376, 380

David, L.P., Forman, W., \& Jones, C. 1991, ApJ, 369, 191

Elbaz, D., Arnaud, M., \& Vangioni-Flam, E. 1995, A\&A, in press

Fabbiano, G., Kim, D.-W., \& Trinchieri, G. 1992, ApJS, 80, 531

Kritsuk, A.G. 1992, A\&A, 261, 78

Loewenstein, M., et al. 1994, ApJ, 436, L75

Loewenstein, M., and Mathews, W.G. 1987, 319, 614

Mathews, W.G., \& Backer, J. 1971, ApJ, 170, 241

Mushotsky, R., et al. 1995, ApJ, in press

Pellegrini, S., \& Fabbiano, C. 1994, ApJ, 429, 105

Renzini, A. 1993, in Panchromatic View of Galaxies, ed. G. Hensler, Ch.

Theis, \& J.S. Gallagher (Gif-sur-Yvette: Editions Frontières), p. 155

Renzini, A., Ciotti, L., D’Ercole, A., \& Pellegrini, S. 1993, ApJ, 419, 52

Renzini, A., Greggio, L., di Serego Alighieri, S., Cappellari, M., Burstein, D., \& Bertola, F. 1995, Nature, 378, 39

Sarazin, C..L., \& White, R.E.III 1987, ApJ, 320, 32

Sarazin, (..L., \& Ashe, C.A. 1989, Ap.J, 345, 22 


\section{Discussion}

Forbes: Does mass drop out of the cooling flow at large radii in your model? Renzini: In the Ciotti et al. (1991) models no distributed mass sink was included. Such an inclusion is mathematically trivial, yet physically ad hoc at this stage. In any event, even models with distributed mass sink cannot explain the distribution of $L_{X}$ vs $L_{B}$ that I have shown.

Elbaz: 1) Concerning the iron enrichment in the intracluster medium and the discrimination between a SNla and a SNII origin, you did not mention the observed Si/Fe ratio by Mushowski with ASCA. 2) Since about half of the Fe mass in clusters is in the ICM and the other half in the galaxies themsemves, one may invoke a common origin and link it to the observed overabundance of $\mathrm{Mg}$ over $\mathrm{Fe}$ in E's (see poster from Barbuy et al. ).

Renzini: 1) Yes, I had a transparency on that but flipped over for lack of time. I will mention the ASCA result in the paper for the proceedings. 2) I don't think that the iron share between galaxies and the ICM can discriminate between a SNIa or a SNII origin. The crucial test is instead in the elemental ratios.

Fabbiano: 1) You said that the Ciotti \& Ostriker model can explain the scatter in $L_{X}$ for a given $L_{B}$. However, the [average $L_{X}-L_{B}$ ] correlation points to the potential also, so the $\mathrm{C \& O}$ effect cannot be all. 2) Is the $\mathrm{C} \& \mathrm{O}$ QSO duty cycle consistent with the fraction of QSOs vs galaxies?

Renzini: 1) I think the average trend of $L_{X}$ with $L_{B}$ is the easiest to reproduce. As I said, ( $\& O$ models jump up and down between $L_{i n}$ and $L_{d i s c r}$, so naturally account also for the observed slope. 2) It seems to me that a duty cycle $\sim 10^{-3}$ is broadly consistent with the QSO/AGN frequency at low $z$. doesn't it?

Ciotti: We expect the duty cycle to depend a lot on the galaxy parameters (especially luminosity), but so far we have not explored the whole parameter space.

Djorgovski: If the mechanism you describe is important for the fueling of quasars, how can you explain the very rapid evolution of the number density of quasars?

Renzini: Probably fueling central BHs from a hot ISM is not the most common way to activate QSOs. The observed peak of the QSO number density at $z=2$ and beyond must have a very different origin, probably intimately connected to the very formation of galactic spheroids. Central cooling catastrophes in ellipticals would just relate to the tail of the QSO density - redshift distribution. Moreover, depending on how much SNla heating is provided by nature, only some among the most massive ellipticals might have already experienced a central cooling catastrophe at late epochs. 